\title{
Publisher Correction: Topological optical differentiator
}

Tengfeng Zhu, Cheng Guo (1), Junyi Huang, Haiwen Wang, Meir Orenstein, Zhichao Ruan (1) \& Shanhui Fan (1)

Correction to: Nature Communications https://doi.org/10.1038/s41467-021-20972-4, published online 29 January 2021.

The original PDF version of this Article contained errors in Equations 3, which incorrectly read:

$$
\mathbf{e}_{\text {out }} \mathbf{R}\left(k_{x}=0, k_{y}=0\right) \mathbf{e}_{\text {in }}=0
$$

The correct form of Eq. (3) is:

$$
\mathbf{e}_{\text {out }}^{\dagger} \mathbf{R}\left(k_{x}=0, k_{y}=0\right) \mathbf{e}_{\text {in }}=0
$$

The PDF version also contained an error in the inline equation at the end of the first paragraph of the first Results' subsection, which incorrectly read:

$$
r\left(k_{x}, k_{y}\right)=\mathbf{e}_{\text {out }} \mathbf{R}\left(k_{x}, k_{y}\right) \mathbf{e}_{\text {in }}
$$

instead of

$$
r\left(k_{x}, k_{y}\right)=\mathbf{e}_{\text {out }}^{\dagger} \mathbf{R}\left(k_{x}, k_{y}\right) \mathbf{e}_{\text {in }}
$$

Both in the PDF and html versions of the article, at the end of the first paragraph of the first Results' subsection, in Equation 2 and in the inline equations after the sentence "The entire reflection process is then described by...", the tilde notations should be exactly above the symbols "S"

Both in the PDF and html versions of the article, at the end of the first paragraph of the first Results' subsection, the inline equation in the sentence starting with "which shows that the zero of..." should read:

$$
r\left(k_{x}, k_{y}\right)
$$

rather than

$$
r t\left(k_{x}, k_{y}\right)
$$

Both the pdf and the html have been updated to include these corrections.

Published online: 07 April 2021

(c) Open Access This article is licensed under a Creative Commons Attribution 4.0 International License, which permits use, sharing, adaptation, distribution and reproduction in any medium or format, as long as you give appropriate credit to the original author(s) and the source, provide a link to the Creative Commons license,

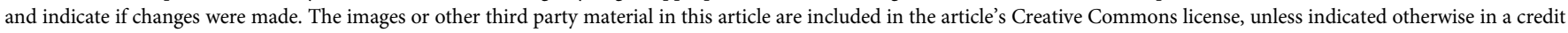
line to the material. If material is not included in the article's Creative Commons license and your intended use is not permitted by statutory regulation or exceeds the permitted use, you will need to obtain permission directly from the copyright holder. To view a copy of this license, visit http://creativecommons.org/licenses/by/4.0/.

(C) The Author(s) 2021 